\title{
Pure Anomic Aphasia Caused by a Subcortical Hemorrhage in the Left Temporo-parieto-occipital Lobe
}

\author{
Masanaka Takeda, Hisao Tachibana, Naomi Shibuya, Yatuka NaKajima, Bungo Okuda, \\ Minoru SugitA and Harumi TANAKA*
}

\begin{abstract}
There have been few case reports of pure anomic aphasia and the underlying mechanism remains to be clarified. We report a patient in whom pure anomic aphasia was caused by subcortical hemorrhage in the left temporo-parieto-occipital lobe. Based on magnetic resonance images and cerebral blood flow imaging, the structural lesion underlying the pure anomic aphasia was thought to be located at the left temporo-occipital junction.

(Internal Medicine 38: 293-295, 1999)
\end{abstract}

Key words: left temporo-occipital junction, regional cerebral blood flow

\section{Introduction}

Anomic aphasia, also called anomia or amnesic aphasia, is characterized by word-finding difficulties in the absence of major syntactic impairment. However, little agreement exists as to which variations should be classified as anomic aphasia, because most patients with anomic aphasia also show impaired comprehension of spoken language to a varying extent (1). Benson (2) first discussed pure anomic aphasia, and proposed that pure anomic aphasia with no other disturbance of output and no problems in comprehension, repetition, reading, or writing, should be called word selection anomia. Very rare cases of pure anomic aphasia have been reported. We describe a patient with pure anomic aphasia caused by a subcortical hemorrhage at the left temporo-parieto-occipital junction. To our knowledge among reported cases known to us, this is the first report in which the pure anomic aphasia occurred from a subcortical hemorrhage.

\section{Case Report}

A 53-year-old right-handed man with a history of hypertension suddenly developed headache and noted difficulty in speaking and a right-sided visual field defect. He was admitted to our hospital on the next day. On admission, the general physical examination was normal; his blood pressure was 150/90 $\mathrm{mmHg}$. Neurologic examination revealed a right homonymous hemianopsia and aphasia with agraphia for Kanji letters. Laboratory test findings included a fasting blood sugar of $231 \mathrm{mg} / \mathrm{dl}$ and a hemoglobin $\mathrm{Al}_{\mathrm{C}}$ concentration of $9.5 \%$. Cranial computed tomography on admission showed a large hematoma in the left temporo-parieto-occipital region (Fig. 1). Surgical evacuation of the hematoma was performed successfully 5 days after admission. Postoperative computed tomography showed complete disappearance of the hematoma, and the right-sided visual field defect was resolved. However, the disturbance of language persisted. Sagittal T1-weighted magetic resonance imaging (MRI) 1 month after surgery demonstrated a low intensity extending from the left middle temporal gyrus to occipital lobe but the superior temporal gyrus and posterior parietal cortex were spared (Fig. 2). A three-dimensional surface display (3) generated from N-isopropyl-p[ $\left.{ }^{123} \mathrm{I}\right]$-iodoamphetamine ( ${ }^{123} \mathrm{I}$-IMP) single-photon emission computed tomography (SPECT) revealed cortical hypoperfusion at the left temporo-parieto-occipital junction (Fig. 3).

Two months after the hemorrhage, the patient's language abilities were assessed with the Japanese version of the Standard Language Test of Aphasia (4; Table 1). His oral expression was fluent without paraphasia, and articulation was fully normal. The information content of speech was limited, reflecting mainly a severe impairment in retrieving object names. Auditory comprehension was unimpaired, and the ability to repeat words was excellent. Object naming was severely impaired, resulting in frequent empty speech and circumlocutions. A verbal description of an object or a circumlocutory response was often substituted for the missing word. For example, when shown a picture of a horse, the patient said, "This is the thing that I can see running on television on Sunday or Saturday."

From the Fifth Department of Internal Medicine, Hyogo College of Medicine, Nishinomiya and *the Department of Rehabilitation, Hyogo College of Medicine, Nishinomiya

Received for publication June 30, 1998; Accepted for publication November 16, 1998

Reprint requests should be addressed to Dr. Masanaka Takeda, the Fifth Department of Internal Medicine, Hyogo College of Medicine, 1-1 Mukogawa-cho, Nishinomiya, Hyogo 663-8501 


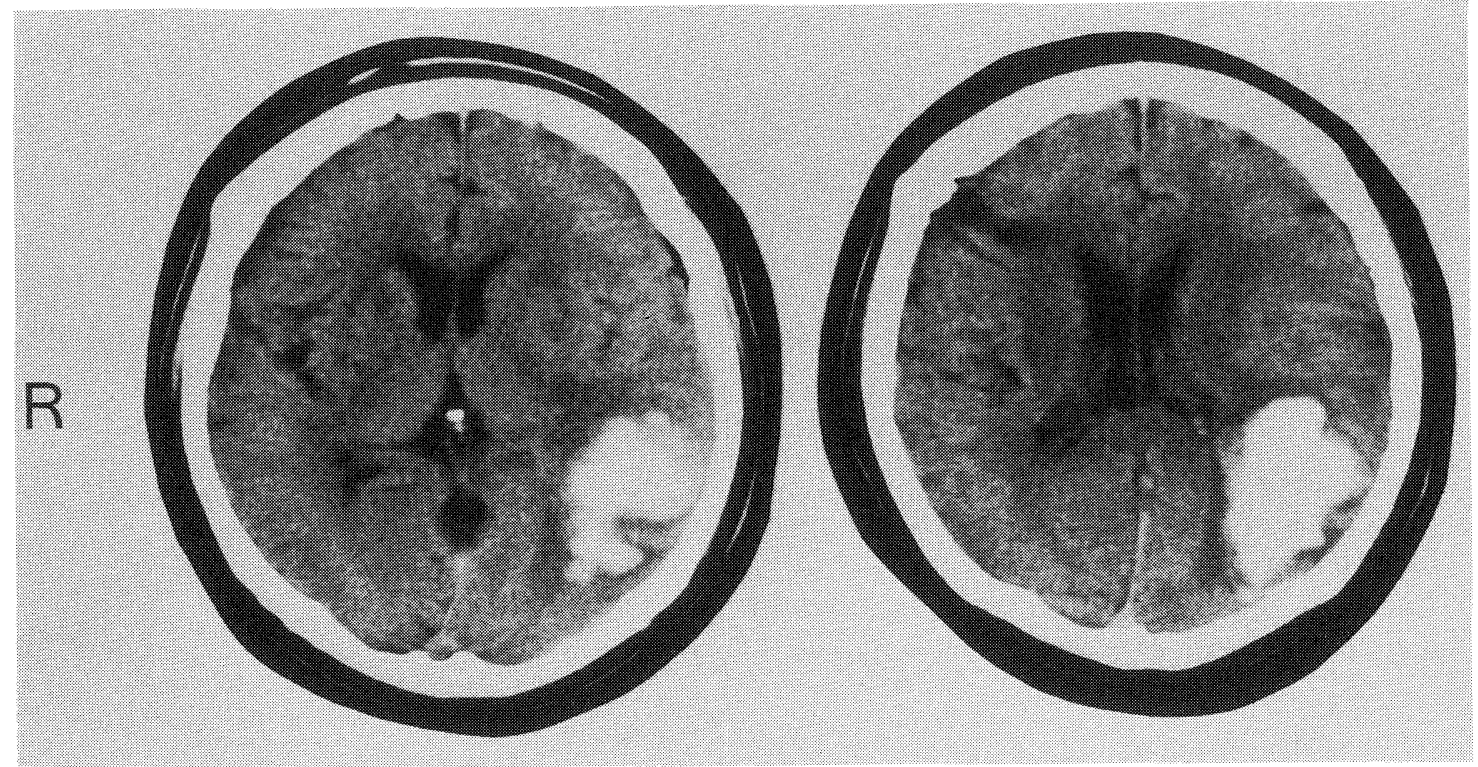

Figure 1. CT scan showing a large hematoma in the left temporo-parieto-occipital lobe.

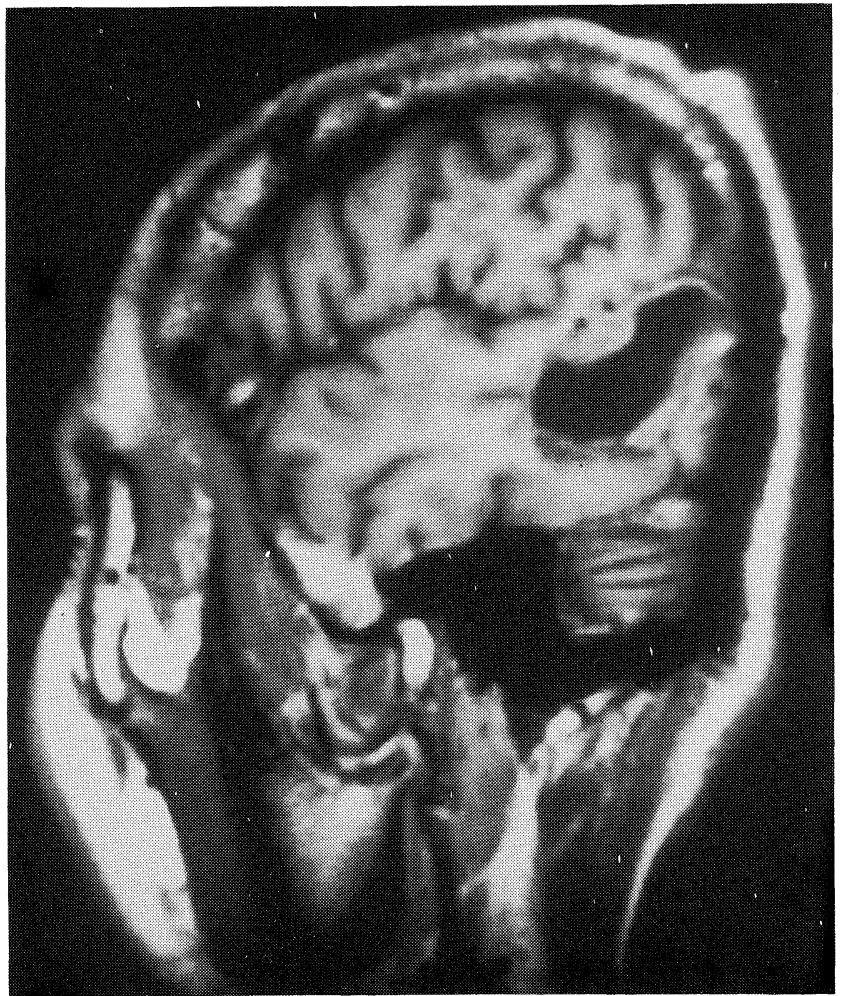

Figure 2. Sagittal T1-weighted MRI showing an extensive area of increased signal intensity from the left middle temporal gyrus to occipital lobe.

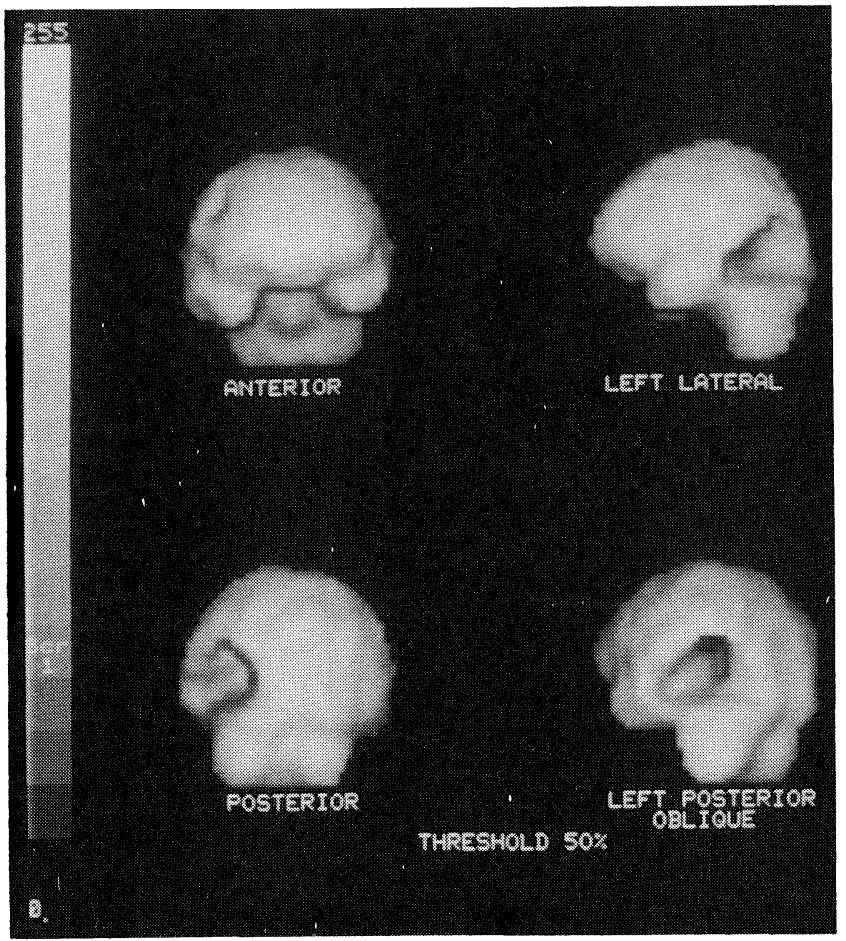

Figure 3. A three-dimensional surface display generated from ${ }^{123}$ I-IMP SPECT $(50 \%$ threshold value of the global maximum counts) showing hypoperfusion in the left temporo-parieto-occipital region. 
Table 1 Results of Speech and Language Evaluations

\begin{tabular}{|c|c|c|}
\hline Types & Tasks & Score* \\
\hline \multirow[t]{3}{*}{ Auditory comprehension } & words & 10 \\
\hline & short sentences & 10 \\
\hline & oral commands & 10 \\
\hline \multirow[t]{4}{*}{ Visual comprehension } & Kanji-words & 10 \\
\hline & Kana-words & 10 \\
\hline & short sentences & 10 \\
\hline & written commands & 9 \\
\hline \multirow[t]{4}{*}{ Speaking } & naming (nouns) & 6 \\
\hline & naming (vervs) & 10 \\
\hline & listing words & 6 \\
\hline & narrative speaking & 8 \\
\hline \multirow[t]{3}{*}{ Written confrontation } & Kanji-words & 6 \\
\hline & Kana-words & 8 \\
\hline & narrative writing & 10 \\
\hline \multirow[t]{2}{*}{ Calculation } &,+- & 10 \\
\hline & $\times, \div$ & 10 \\
\hline \multirow[t]{2}{*}{ Repetition } & words & 10 \\
\hline & sentences & 9 \\
\hline \multirow[t]{3}{*}{ Oral reading } & Kanji-words & 10 \\
\hline & Kana-words & 10 \\
\hline & short sentences & 10 \\
\hline \multirow[t]{3}{*}{ Writing to dictation } & Kanji-words & 6 \\
\hline & Kana-words & 10 \\
\hline & short sentences & 10 \\
\hline \multirow[t]{3}{*}{ Individual Kana character } & comprehension & 9 \\
\hline & oral rading & 10 \\
\hline & dictation & 10 \\
\hline
\end{tabular}

*The perfect score for all types of tests was 10 .

Reading was flawless, while naming of objects in writing was as severely impaired as oral naming, especially when using Kanji letters. No acalculia or apraxia was demonstrated.

\section{Discussion}

The principal feature of our patient's language function was difficulty in confrontation naming with the absence of disturbance of speech output, comprehension, or repetition. This case was consistent with pure anomic aphasia since comprehension was full, although writing of Kanji letters was impaired. Some cases of anomic aphasia without an auditory comprehension disorder have been reported $(2,5-8)$. There has been considerable disagreement concerning the lesion site responsible for pure anomic aphasia (2). The previously reported anatomical lesions in anomic aphasia include the left superior temporal and posterior parietal lobes, and frequently involve Wernicke's area or the angular gyrus (9-11). In the present case, MRI showed a lesion involving the left posterior temporal and occipital lobes but not the superior temporal gyrus or posterior parietal cortex. In the present patient, such sparing of Wernicke's area and the angular gyrus correlates with preserved auditory and lexical comprehension. SPECT revealed hypoperfusion restricted to the left temporo-occipital junction. Kezuka and Kawamura (6) reported a case, with a lesion in the left inferior temporal lobe which extended to the occipital cortex. The damage to the left temporo-occipital junction of their case overlapped the findings of the present case.

In addition to anomic aphasia, the present patient had impairment of writing Kanji letters. The middle and inferior temporal gyri have been reported to contribute to reading and writing Kanji letters (12) based on a case where the main lesion site was the posterior part of the middle temporal gyrus extending to the temporo-occipital junction. In our case, a subcortical hemorrhage caused a lesion which was restricted to the posterior part of the middle temporal gyrus and the adjacent occipital lobe, resulting in pure anomic aphasia with Kanji agraphia.

\section{References}

1) Benson DF, Ardila A. Aphasia. A Clinical Perspective. University of Oxford Press, New York, 1996, pp. 252-261.

2) Benson DF. Neurologic correlates of anomia (vol 4). in: Studies in Neurolinguistics, Whitaker H, Whitaker HA, Eds. Academic Press, New York, 1979, pp. 293-328.

3) Tachibana H, Kawabata K, Tomino Y, Sugita M, Fukuchi M. Brain perfusion imaging in Parkinson's disease and Alzheimer's disease demonstrated by three-dimensional surface display with ${ }^{123}$ I-iodoamphetamine. Dementia 4: 334-341, 1993.

4) Takeda K. Standard language test of aphasia: detailed description of construction of aphasia test in Japanese (in Japanese). Adv Neurol Sci (Tokyo) 21: 1002-1013, 1977.

5) Gainotti G, Silveri MC, Villa G, Miceli G. Anomia with and without lexical comprehension disorders. Brain Lang 29: 18-33, 1986.

6) Kezuka M, Kawamura M. Naming difficulty associated with the left inferior temporal lesion. Shinkei Shinrigaku (Jpn J Neuropsychologia) 10: 26-31, 1994 (Abstract in English).

7) Miozzo A, Soardi M, Cappa SF. Pure anomia with spared action naming due to a left temporal lesion. Neuropsychologia 32: 1101-1109, 1994.

8) Raymer AM, Foundas AL, Maher LM, et al. Cognitive neuropsychological analysis and neuroanatomic correlates in a case of acute anomia. Brain Lang 58: 137-156, 1997.

9) Miceli G, Silveri MC, Nocentini U, et al. Pattern of dissociations in comprehension and production of nouns and verbs. Aphasiology 2: 351-358, 1988.

10) Damasio A, Damasio H. Lesion Analysis in Neuropsychology. University of Oxford Press, 1989, pp. 122-125.

11) Damasio H. Neuroanatomical correlates of the aphasia. in: Acquired Aphasia, Eds. Sarno MT, 2nd edition, Academic Press, 1991, pp. 52-53.

12) Iwata M. Kanji versus Kana. Neuropsychological correlates of the Japanese writing system. Trends Neurosci 7: 290-293, 1984. 